\title{
Pediatric care report of spinal cord injury without radiographic abnormality (SCIWORA): case report and literature review
}

\author{
A Ergun*,1 and W Oder ${ }^{1}$ \\ ${ }^{1}$ Rehabilitation Center for Head Trauma Patients Meidling, AUVA, Vienna, Austria
}

Study design: Case report of SCIWORA following trivial trauma in childhood and review of the literature.

Objectives: To describe a case of 'spinal cord injuries without radiographic abnormality (SCIWORA)', which was diagnosed 2 years after the event, and define its relevance from a differential diagnostic and medico-legal viewpoint.

Setting: An Austrian neuro-traumatological rehabilitation and workers' insurance appraisal center.

Methods: A 12-year-old patient is presented who suffered an ischemic spinal lesion of unknown origin on her way home from school. The patient was reinvestigated as part of an insurance appraisal 2 years later. The aim of this investigation was to elucidate the origin of the spinal cord lesion, in particular, whether it was because of accidental trauma or because of a preexisting condition (eg vascular malformation).

Results: SCIWORA due to a trivial trauma was diagnosed during the reinvestigation. This was of importance for the patient because of accident insurance coverage.

Conclusion: In children developing a quadroparesis following a minor trauma, spinal cord injury without radiographic abnormality must be considered in the differental diagnosis.

Spinal Cord (2003) 41, 249-253. doi:10.1038/sj.sc.3101442

Keywords: SCIWORA; anterior spinal artery infarction; pediatric traumatic myelopathy

\section{Introduction}

Spinal cord injuries without radiographic abnormality (SCIWORA), ${ }^{1-5}$ also known as the "pediatric syndrome of traumatic myelopathy without demonstrable vertebral injury' in the literature, ${ }^{6}$ are a primarily pediatric syndrome. Turgut ${ }^{7}$ described its incidence in $13 \%$ of the 82 children he treated with spinal cord and/or vertebral column injury. Pang, ${ }^{2}$ on the other hand, reported its incidence at $66.7 \%$.

There are few case reports on children suffering from SCIWORA, which demonstrate that even a very minor trauma can cause severe spinal cord lesions. Linssen ${ }^{8}$ reported an 11-year-old boy, who held his neck in hyperextension for $30 \mathrm{~min}$ while playing on a swing, and $1 \mathrm{~h}$ later developed an incomplete spinal cord syndrome below $\mathrm{C} 4 / \mathrm{C} 5$. Cheshire ${ }^{6}$ described a $6 \frac{1}{2}$-year-old girl, who developed a Th11 syndrome after a backward somersault from her bed to the floor, a 3-year-old boy, who tripped and fell while playing, developing an incomplete spinal cord injury below $\mathrm{C} 4$ a few hours later, and a boy aged 2 with a complete spinal cord

*Correspondence: A Ergun, Rehabilitation Center for Head Trauma Patients Meidling, AUVA, Koeglergasse 2a, A-1120 Vienna, Austria lesion below $\mathrm{C} 5$ who had run into a metal table some hours before. Similarly, Riviello ${ }^{9}$ saw a 3 -year-old girl who did a somersault on her couch, fell on her back and $1 \mathrm{~h}$ later developed a progressive quadriparesis. In another case, a 2-year-old girl fell onto a carpeted floor while 'riding' on the arms of her couch. After $2 \mathrm{~h}$ progressive quadriparesis with respiratory failure started.

In the majority of cases, SCIWORA is caused by hyperextension or flexion injuries due to characteristic anatomic differences of the more elastic and more deformable pediatric spine. ${ }^{2,3,5}$ These flexion-extension injuries may provoke a temporary occlusion of the vertebral arteries or the anterior spinal artery with the result of a spinal cord infarction. ${ }^{10}$

SCIWORA has also been described in adults, although a different pathogenesis has been postulated. Gupta et al ${ }^{11}$ described 15 adults with SCIWORA. These were nearly $10 \%$ of all spinal cord injuries seen in 1 year. A disc prolapse was seen in six patients, intramedullary hemotoma/contusion in four patients, cord edema in four patients, and multiple disc prolapses with associated intramedullary hemotoma in one patient. 
In the differential diagnosis in pediatric SCIWORA, a nontraumatic thrombosis of the vertebral arteries due to embolism, vascular malformation, and an acute or chronic inflammation of the spinal cord have to be ruled out primarily.

In Austria, every employee who suffers an accident that is related to his/her job is covered by law by a special insurance, the 'Allgemeine Unfallversicherung'. The accident is defined as a sudden and timewise limited event, which either takes place at work/school or on the way to or from work/school. Treatment and rehabilitation costs as well as financial compensation are offered when necessary, the worsening of a preexisting disease or abnormality which occurs unrelated to work is, however, not covered. The financial compensation is meant as a substitute for any loss of income due to functional deficits that did not recover from the accident. The severity of deficits is estimated according to fixed guidelines, which are also the basis for the amount of compensation.

\section{Case report}

A healthy 12-year-old girl slipped on an icy path in the woods on her way home from a volleyball match at school.

At 2 years after the injury, the patient was reexamined for insurance reasons. She reported that she had suddenly toppled over and fell on her knees. She could not get up again because of a weakness in her legs. She had no pain. Conflicting hospital reports from the time of admission exist; one of them stipulates that the patient fell because of a prior, sudden weakness in both of her legs, the others stating that the weakness was a result of her fall.

All previous hospital reports, starting from the first day of illness until the present examination, were available. On admission to the hospital $2 \mathrm{~h}$ after onset, an incomplete spinal cord transection at cervical $\mathrm{C} 6$ was diagnosed with paraplegia and weakness in both upper limbs, particularly affecting hands and fingers and impairing bladder function. No signs of external wounds were found. An X-ray of the cervical and thoracal spine showed no traumatic lesions. A cerebral and complete spinal cord magnetic resonance tomography (MRI) with contrast performed 2 days following the accident revealed a hyperintense lesion extending from the segments C6 to thoracic (Th)7 in the ventral part of the spinal cord, which was described as a probable infarction of the anterior spinal artery radiologically. Further investigation, including angiography with selective representation of both vertebral arteries, the radiculo-medullar branches and the trunk of the anterior spinal artery, as well as a cardiac investigation including ECG and echocardiography, visually evoked potentials, laboratory tests with particular emphasis on coagulopathies and a spinal tap were unremarkable.

An acute or chronic inflammatory lesion could not, however, be fully excluded and therapy with steroids was initiated through the first 6 weeks (intravenous

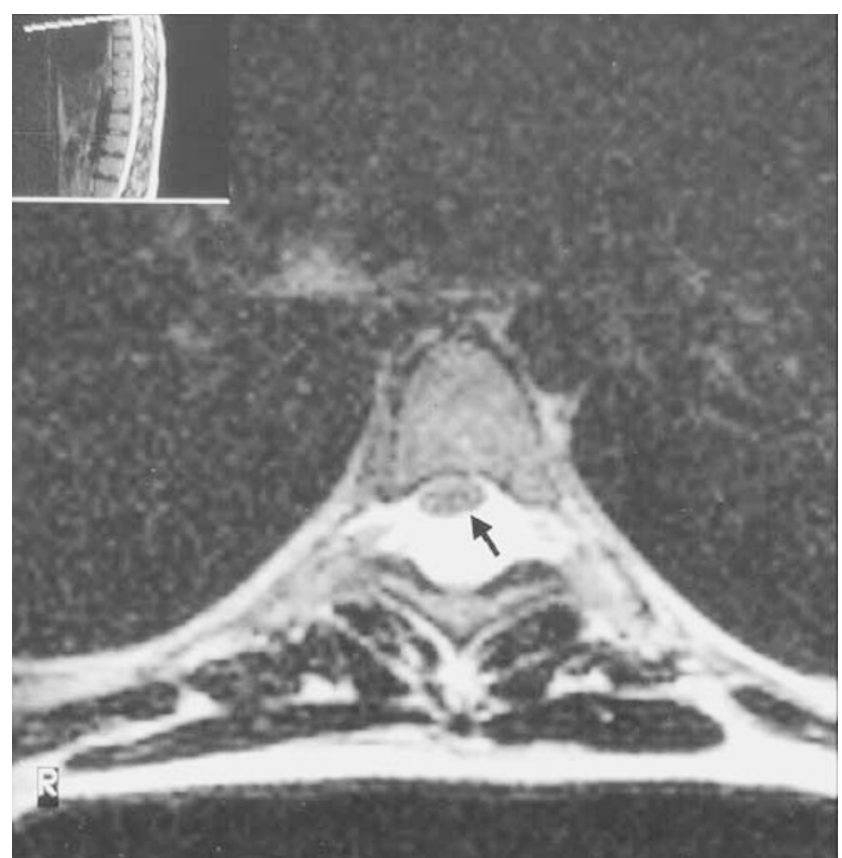

Figure 1 MRI C5-C6 2 years post-trauma, median sagittal diameter of myelon $5 \mathrm{~mm}$.

dexamethasone for 10 days followed by oral prednisolone with slow dosage reduction). Remission started slowly 3 weeks after onset with discrete active motility in the left leg and returning bladder function.

At 5 months following the trauma, the patient was able to walk approximately $100 \mathrm{~m}$ without any support. Bladder function improved, but was still insufficient. At that time, the patient was still diagnosed as incomplete spinal cord transection of the lower cervical and upper thoracic spinal cord of unknown origin.

At 2 years following the accident, further recovery had occurred. The patient was referred to a special rehabilitation department as part of an insurance appraisal. Neurologically, there was a discrete spastic tetraparesis with mild spasticity, accentuated on the right side with pyramidal signs in all four limbs and slightly reduced force in both lower limbs, accentuated on the right side. The right leg dragged slightly behind when walking.

The MRI with contrast to the brain and the spinal cord showed a rather thin spinal cord in the segments C5 and C6 with a median-sagittal diameter of $5 \mathrm{~mm}$ (Figure 1), the spinal cord increasing caudally with a diameter of $8 \mathrm{~mm}$ at segments Th11 and Th12 (Figure 2).

\section{Discussion}

In Austria, injuries that occur at or on the way to and from work/school are covered by an insurance that facilitates a lifelong compensation for any resulting permanent deficits from the accident and also assists in the integration of the individual into the labor process. Therefore, it was of enormous importance for the future of the child to elucidate the origin of the spinal cord 


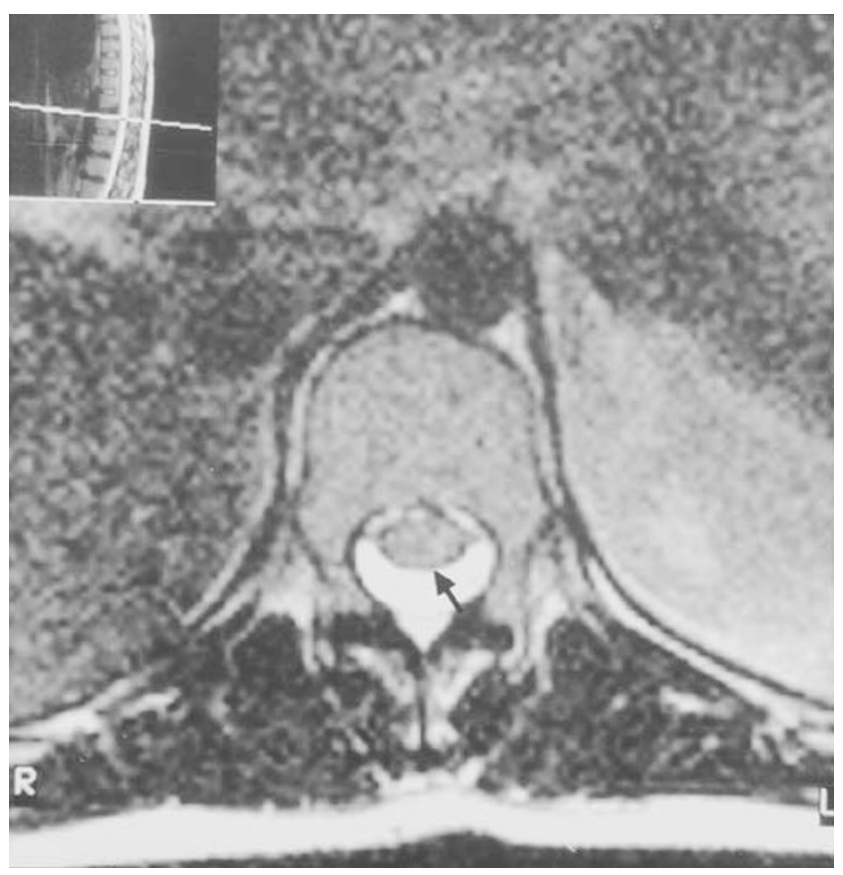

Figure 2 MRI Th11-Th12 2 years post-trauma, median sagittal diameter of myelon $8 \mathrm{~mm}$.

lesion, in particular, whether it was due to accidental trauma or due to a pre-existing condition (eg vascular malformation).

In the acute phase of injury, the patient had been diagnosed with an incomplete spinal cord transection at cervical C6 of unknown origin. At the time of reinvestigation 2 years later, spinal cord injury without radiographic abnormality (SCIWORA), ${ }^{1-5}$ also known as the 'pediatric syndrome of traumatic myelopathy without demonstrable vertebral injury' ${ }^{6}$ was diagnosed.

The 12-year-old patient had suffered ischemia in the ventral part of the spinal cord C6-Th7, corresponding to an anterior spinal artery infarction. The anterior spinal artery receives its main blood supply from vessels out of the vertebral arteries primarily following the neural roots $\mathrm{C} 6 / \mathrm{C} 7$ for the branches of the lower cervical cord. The arterial vascularization of the segmental medullary branches to the lower cervical and upper thoracal cord can vary considerably. ${ }^{12}$

Hyperextension may provoke a temporary occlusion or spasm of the vertebral arteries, ${ }^{10}$ the anterior spinal artery or the distal branches of the central sulcus arteries ${ }^{8}$ leading to a spinal cord infarction. ${ }^{10}$

The mechanisms of injury in SCIWORA are in most cases hyperextension, flexion, and to a lesser extent longitudinal distraction, direct crush injury or combinations thereof. ${ }^{2,3,5}$ The pediatric spine is more elastic and therefore more deformable. ${ }^{3}$ During hyperextension, the interlaminar ligaments of the cervical spine bulge forward into the central canal, narrowing the cervical canal by over $50 \%$. Additionally, the spinal cord simultaneously shortens and thickens. Pre-existing spondylosis or congenital stenosis constitutes an aggra- vating factor. ${ }^{3}$ The smaller size of the cervical spinal canal in girls may account for the female predominance of SCIWORA following trivial trauma. ${ }^{9}$

The susceptibility of the cervical column to flexion injuries is increased because firstly, the planes of the facet joints are more horizontal and secondly, the anterior portion of the vertebral bodies are wedged forward. These findings are most prominent in the upper three to four segments of the cervical spine facilitating anterior slipping between adjacent bodies with spinal injuries resulting in this region in young children. The uncinate processes cannot withstand flexion-rotation forces as they are flat. The proportionately heavy head and relatively undeveloped musculature of the infant neck are highly susceptible to flexion-extension injuries. $^{3}$

Paresis due to SCIWORA may start immediately after trauma or may present itself with a delayed onset from a few minutes up to $24 \mathrm{~h}^{2,6}$ and even up to 4 days. ${ }^{5}$ Once paresis begins it progresses rapidly. ${ }^{3}$ Ruge ${ }^{4}$ reported a delayed onset in $52 \%$ of his cases with SCIWORA. Some patients noticed transient neurological symptoms at the time of injury. ${ }^{3} \mathrm{Pang}^{2}$ saw a delayed onset in 15 of his 55 studied cases with SCIWORA. Nine of these had transient warning symptoms immediately following the trauma. The reason for the 'latent' period preceding severe neurological damage has been attributed to repeated movements of the spine following the original subluxation, which again causes multiple repetitive insults to the cord at the site of the original injury causing progressive destruction of neural tissues. ${ }^{3}$

In the case presented, paralysis might have started immediately after slipping, when the 12-year-old most probably performed a hyperextension movement with the cervical column. However, the symptoms could have equally started with a latency of approximately $1-2 \mathrm{~h}$, the trauma having taken place while playing volleyball. The girl then fell because of the beginning weakness in her feet, as was documented in one of the clinical reports at the time of admission.

The characteristic anatomical features of the young spine gradually approach adult status by the age of 16-18 years with the cervical spine gaining resistance against flexion injuries. Flexion forces then predominantly produce fracture and subluxation, respectively. ${ }^{3}$ In younger children, damage selectively affects the cervical spine above $\mathrm{C} 3$, while with increasing age, damage is sustained lower down the cervical spine. ${ }^{5}$

Younger children suffer severe lesions more frequently than elder ones. The level of the neurological lesion influences the prognosis with higher cervical injuries being worse than thoracic lesions. ${ }^{3}$ An initially severe neural injury is almost always associated with a poor prognosis. ${ }^{2}$ Most children with complete and severe lesions do not recover. The best outcome was seen in patients with mild deficits initially. ${ }^{3}$

Pang $^{2}$ studied 55 children with SCIWORA aged between 6 months and 16 years. In all, 10 had upper cervical (C1-C4), 33 had lower cervical (C5-C8) and 12 
thoracic cord injuries. In total, 22 children had complete or severe neurologic injuries, with a significantly high percentage of upper cervical lesions. All but one of the patients were younger than 8 years old. In all, 33 children suffered from mild lesions with 24 of them older than 8 years.

In the patient described, the spinal cord syndrome, which was incomplete and located in the lower cervical and upper thoracal spine, shows similarities with the findings in the literature concerning age and location. Our patient showed a very good, although incomplete recovery with a minimal spastic tetraparesis accentuated on the right 2 years after the trauma.

Concerning the differential diagnosis in SCIWORA, the syndrome of acute cervical central cord injury following hyperextension as described by Schneider ${ }^{13}$ must be excluded. It is characterized by a disproportionately greater motor impairment of the upper than of the lower extremities, bladder dysfunction and varying degrees of sensory loss below the level of the lesion. The syndrome was described following hyperextension without fracture or fracture dislocation only in elderly adults. In younger adults, bleeding, contusion or concussion in the cervical central cord has been caused by severe cervical compression fractures and cervical fracture dislocations.

A nontraumatic thrombosis of the vertebral artery, in particular embolism because of cardiological diseases such as endocarditis, arrhythmia, a persistent foramen ovale and thrombosis due to arteritis or coagulopathies has to be kept in mind, as well. It has been ruled out in the described patient ${ }^{14}$ by an extensive cardiovascular investigation including ECG and echocardiography and laboratory tests with particular emphasis on coagulopathies, all showing unremarkable results.

In the acute phase, a possible differential diagnosis was an acute or chronic inflammation of the spinal cord. Therfore, at the time of injury as well as at the time of reinvestigation, visually evoked potentials and multiple spinal taps were performed but no pathology was observed. The patient, however, was still treated with steroids, which is the treatment for an acute attack of chronic inflammation, for example, multiple sclerosis ${ }^{15}$ or for SCIWORA. ${ }^{3-9,16}$

Technically inadequate radiographic series of the cervical spine may miss the diagnosis of a cervical spine injury. Views required include a posterioanterior, lateral and odontoid view. The lateral view must include all cervical vertebrae and the C7-Th1 interspace. ${ }^{16}$ Even in the face of a three-view series, cervical spine injury must be suspected in patients with an abnormal neurological exam, high-risk-injury mechanism or a distracting injury. ${ }^{17}$

In the patient presented, SCIWORA was diagnosed at the time of reinvestigation. As a result, the injury was due to an accidental trauma. For the young patient this meant a lifelong financial compensation for her remaining deficits, which in her case were based on $40 \%$ of lowering of working abilities due to spastic tetraparesis and slightly reduced force in both lower limbs, accentuated on the right side. It also meant a fully reimbursed rehabilitation whenever needed, and further assistance in the labor process.

In conclusion, a spinal cord injury without radiographic abnormality has to be kept in mind in children developing a quadroparesis following a minor trauma. In the case presented, the patient received best medical treatment in the acute phase of trauma, although SCIWORA was not diagnosed at the time. The diagnosis of SCIWORA was of enormous importance for the patient described from a legal point of view. The decisive factors in diagnosing SCIWORA 2 years after the event were the availability of all medical reports and a very detailed patient history.

\section{References}

1 Govender S, Charles RW, Rasool MN. Spinal injuries in children. Injury 1990; 21: 403-406.

2 Pang D, Pollack IF. Spinal cord injury without radiographic abnormality in children-the SCIWORA syndrome. J Trauma 1989; 29: 654-664.

3 Pang D, Wilberger JE. Spinal cord injury without radiographic abnormalities in children. J Neurosurg 1982; 57: 114-129.

4 Ruge JR, Sinson GP, McLone DG et al. Pediatric spinal injury: the very young. J Neurosurg 1988; 68: 25-30.

5 Short DJ, Frankel HL, Bergström EMK. Injuries of the spinal cord in children. In: Frankel HL (ed). Handbook of Clinical Neurology, Vol. 17. Elsevier: Amsterdam 1992, pp 233-252.

6 Cheshire DJE. The pediatric syndrome of traumatic myelopathy without demonstrable vertebral injury. Paraplegia 1977; 15: 74-85.

7 Turgut M, Akpinar G, Akalan N et al. Spinal injuries in the pediatric age group: a review of 82 cases of spinal cord injury and vertebral column injuries. Eur Spine J 1996; 5: $148-152$.

8 Linssen WHJP, Praamstra P, Gabreels FJM et al. Vascular insufficiency of the cervical cord due to hyperextension of the spine. Pediatr Neurol 1990; 6: 123-125.

9 Riviello JJ, Marks HG, Faerber EN et al. Delayed cervical central cord syndrome after trivial trauma. Pediatr Emerg Care 1990; 6: 113-117.

10 Ahmann PA, Smith SA, Schwartz JF et al. Spinal cord infarction due to minor trauma in children. Neurology 1975; 25: 301-307.

11 Gupta SK, Rajeev K, Khosla VK et al. Spinal cord injury without radiographic abnormality in adults. Spinal Cord 1999; 37: 726-729.

12 Unterharnscheidt F. Gefäßversorgung des menschlichen Rückenmarks. In: Doerr W, Seifert G, Uehlinger E (eds). Spezielle Pathologische Anatomie 13/VII. Springer: Berlin, Heidelberg 1992, pp 27-45.

13 Schneider RC, Thompson JM, Bebin J. The syndrome of acute central cervical spinal cord injury. $J$ Neurol Neurosurg Psychiatry 1958; 21: 216-227.

14 Unterharnscheidt F. Wirbelsäulen- und Rückenmarksverletzungen im Kleinkindes- und Kindesalter In: Doerr W, Seifert G, Uehlinger E (eds). Spezielle Pathologische Anatomie 13/VII. Springer: Berlin, Heidelberg 1992, pp 571-577. 
15 Brainin $\mathrm{M}$ et al. Changes within the 'normal' cerebral white matter of multiple sclerosis patients during acute attacks and during high-dose cortisone therapy assessed by means of quantitative MRI. J Neurol Neurosurg Psychiatry 1989; 52: $1355-1359$.
16 Graber MA, Kathol M. Cervical spine radiographs in the trauma patient. Am Fam Physician 1999; 59: 331-342.

17 Baker C, Kadish H, Schunk JE. Evaluation of pediatric cervical spine injuries. Am J Emerg Med 1999; 17: 230-234. 